\title{
Research on the Developing Suggestions of Enterprise Competitive Intelligence in Big Data Era
}

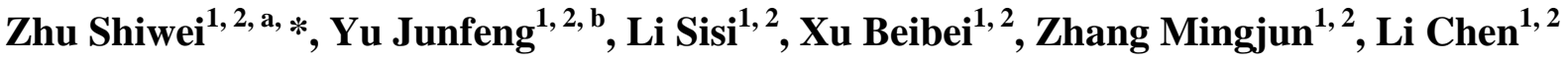 \\ ${ }^{1}$ Information Research Institute of Shandong Academy of Sciences, No. 19, Keyuan Road, Jinan, China \\ ${ }^{2}$ Qilu University of Technology, No. 3501, University Road, Jinan, China \\ a zhusw@sdas.org, byujf@sdas.org
}

Keywords: big data, competitive intelligence, enterprise big data, cloud computing, big data application

\begin{abstract}
With the rapid development of information technology and networks, the big data era is coming, great changes of the data usage in all industries have taken place. On the top of the pyramid, competitive intelligence is bound to be impacted. The article describes the concept and the donation of big data, then deeply analysed the statement of big data brings to competitive intelligence, including the opportunities and challenges. Finally, we propose the strategies and suggestions of enterprise competitive intelligence development, which focusing on competitive intelligence systems, intelligence organization team, resource sharing and collaboration, and intelligence security system constructing.
\end{abstract}

\section{Introduction}

Intelligence researching is the process of extracting valuable information from bulk data and information, and providing related solutions for decision-making, i. e., it's the process of data processing, organization and interpretation, to reveal the potential knowledge, and then turn into usable information. Innovation oriented intelligence analysis is an important subject in area of intelligence and strategic management, and owns a lot of research results ${ }^{[1]}$. It is the key value in intelligence activities to transform information to intelligence. However, facing the big data environment with accumulated information socialization, traditional intelligence analysis based on the experience of simple statistics can no longer abstract semantic content and associated knowledge form vast open source information, which deeply affected the depth of intelligence efficiency ${ }^{[2]}$.

\section{The connotation of big data}

Big data is a term that describes the large volume of data, both structured and unstructured, that inundates a business on a day-to-day basis ${ }^{[3]}$. But it's not the amount of data that's important. The term has been in use since the 1990's, with some giving credit to John Mashey for coining or at least making it popular. Big data is data sets that are so voluminous and complex that traditional data processing applications are inadequate to deal with them ${ }^{[3]}$. There are a number of concepts associated with big data: originally there were 3 concepts volume, variety and velocity. Big data philosophy encompasses unstructured, semi-structured and structured data, however the main focus is on unstructured data. Big data size is a constantly moving target, as for 2012 ranging from a few dozen terabytes to many exabyte of data ${ }^{[4]}$.

\subsection{The characteristics of big data}

Big data can be described by the following characteristics:

Volume, is the quantity of generated and stored data, the size of data determines the value and potential insight, and whether it can be considered big data or not. Volume is a key contributor to the problem of why traditional relational database management systems fail to handle big data. 
Underlying that failure are more complex issues of cost, reliability, long query times, and their inability to handle new sources of unstructured or semi-structured data like text ${ }^{[5]}$.

Variety, is the type and nature of the data, it helps people who analyse that to effectively use the resulting insight. Big data draws from text, images, audio, video, plus it completes missing pieces through data fusion. The variety of big data includes two questions: different types of data, and different source of data. In terms of opportunity, variety is seen by business users as the major focus of new big data initiatives. Companies have been handling large volumes of data for many years and view that process as incremental and business and usual ${ }^{[6]}$.

Velocity, the speed at which the data is generated and processed to meet the demands and challenges that lie in the path of growth and development. Big data is often available in real-time. Velocity is to describe data-in-motion, for example, the steam of readings taken from a sensor or the web log history of page visits and clicks by each visitor to a web site. Consistency and completeness of fast moving streams of data are one concern. Matching them to specific outcome events, a challenge raised under Variety is another. Velocity also incorporates the characteristics of timeliness or latency - is the data being captured at a rate or with a lag time that makes it useful.

Veracity, the data quality of captured data can vary greatly, affecting the accurate analysis. What is the provenance of the data? Does it come from a reliable source?

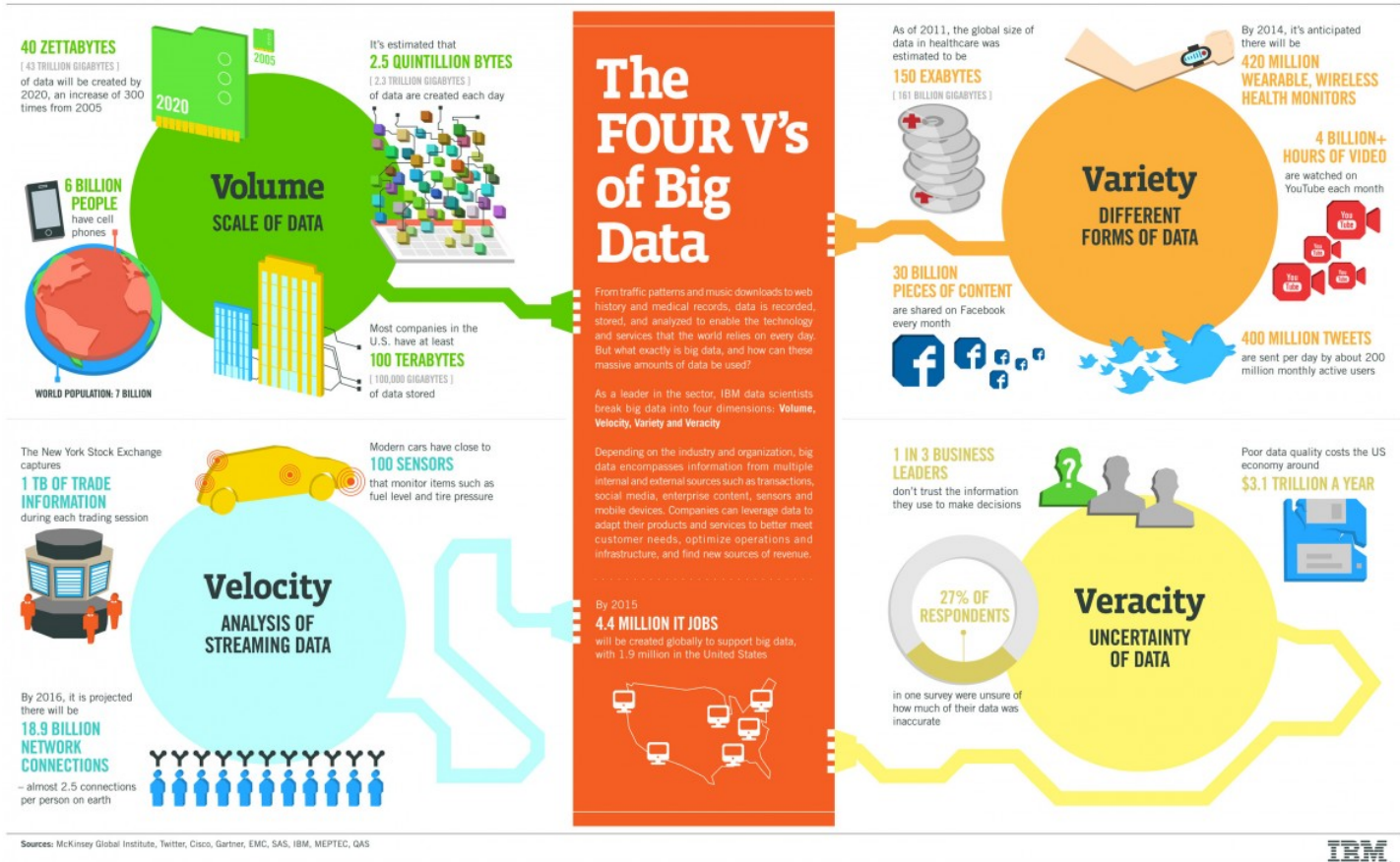

Figure 1 The 4 V's of big data

\subsection{The architecture of big data systems}

Big data repositories have existed in many forms, often built by corporations with a special need. Commercial vendors historically offered parallel database management systems for big data beginning in the 1990's ${ }^{[6]}$. A big data solution typically comprises the following logical layers:

(1) Big data sources: Think in terms of all of the data available for analysis, coming in from all channels. Ask the data scientists in your organization to clarify what data is required to perform the kind of analyses you need. The data will vary in format and origin:

Format: Structured, semi-structured, or unstructured.

Velocity and volume: The speed that data arrives and the rate at which it's delivered varies according to data source.

(2) Data massaging and store layer: This layer is responsible for acquiring data from the data sources and, if necessary, converting it to a format that suits how the data is to be analysed.

(3) Analysis layer: The analysis layer reads the data digested by the data massaging and store layer. In some cases, the analysis layer accesses the data directly from the data source. 
(4) Consumption layer: This layer consumes the output provided by the analysis layer. The consumers can be visualization applications, human beings, business processes, or services.

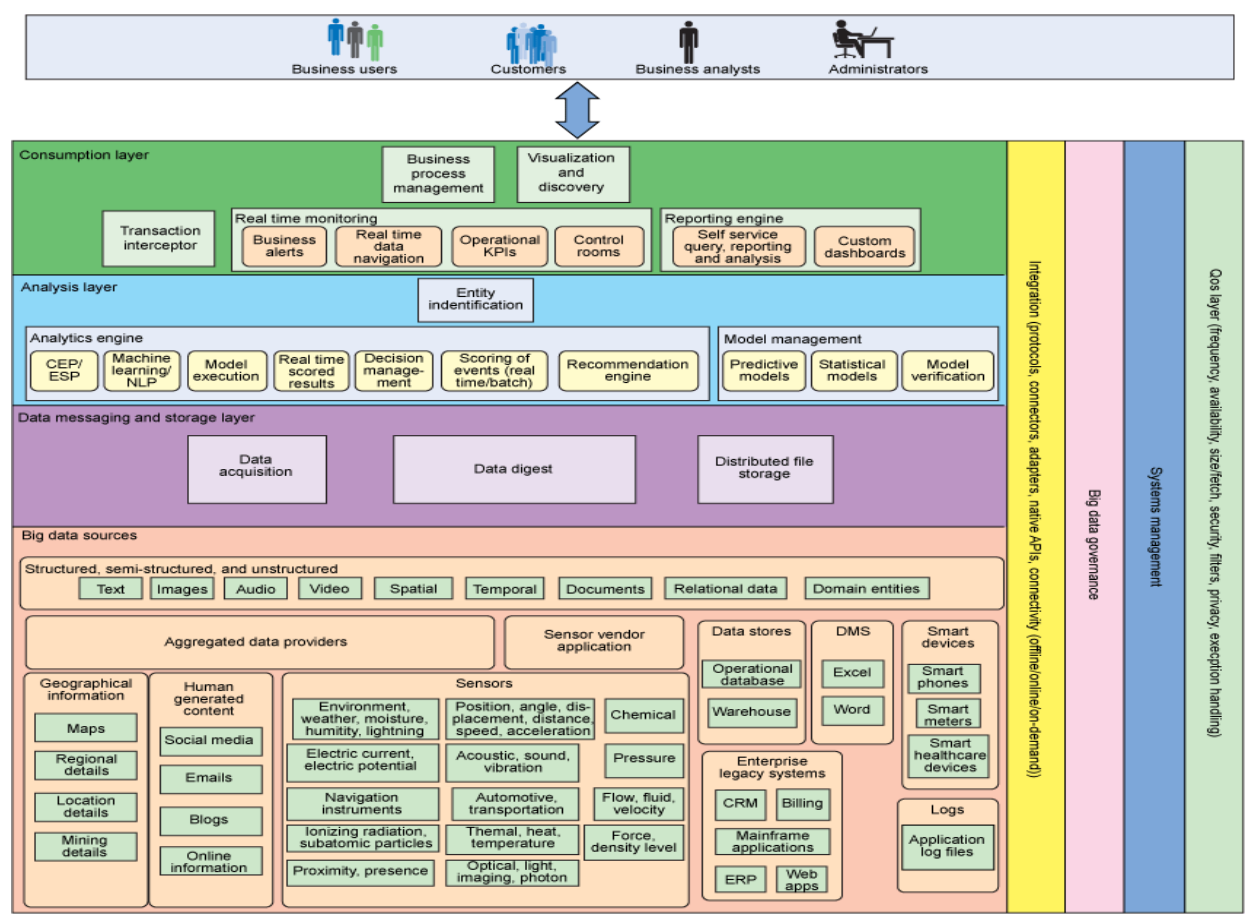

Figure 2 Components by logical and vertical layer

\section{Competitive Intelligence (CI) in Big Data Era}

\subsection{Big data applications in competitive intelligence}

There are a large number of stories and analysis about the big data on domestic leading and local journals, big data analysis tools and applications have been the research hotspots in intelligence science and other related domains and the new research achievement and challenges have involved, which contains several critical techniques including data mining, DFS, visualization etc.

However, the related techniques and tools in intelligence science domain are limited. There is no doubt that big data techniques provide the new way for information institute to change the knowledge service pattern, to modify the business including the knowledge management pattern, the cooperating and interactive pattern, dynamically supervising knowledge service flow etc. We can predict that real time access, accurate analysis, deep mining from massive, complicated, multiple structural data can be applied in the real world. The big data analysis will follow the commercial pattern, while the core infrastructure will be applied for the service and application ${ }^{[10]}$.

\subsection{The influence of big data on CI}

The reliability of intelligence on comprehensive data has been improved by big data analysis. From the prospect of data source, big data contains three major types, including transaction data, interactive data and perception data. Specifically, transaction data, stored in SQL database, is the business data from the ERP, SCM, CRM and Web transaction system of companies. Interactive data is mainly generated from social network, such as Weibo, Facebook, Twitter, Web blog, Clickstream data, email etc. Perception data, mainly collected from Internet of Things, such as sensors, RFID, GPS etc, indicates the awareness of surroundings in the real world. The data which is generated from various data source denotes the different aspects of competitors, environment and the sources, and provides sufficient information for apperceiving the trend of competition among companies and hot spots, the weakness and strength of companies ${ }^{[10]}$.

The accuracy of intelligence on socialized data has been dramatically increased. One of significant trends in big data era is the data socialization, which can be observed in the related records from networks, such as blog, game community, Weibo, internet, mobile network and 
Internet of Things etc. Data socialization facilitates companies to be close to customers and to observe their behaviours, and supplies opportunities for workers to collect first-hand information related to users, which can derive accurate and predictive knowledge for competition. The new social applications such as Twitter, Facebook, Weibo etc generate socialized data, which improve the reliability and accuracy of intelligence.

The applicability of intelligence on real-time series data has been obviously enhanced by big data technology. Currently the centre of gravity of internet has gradually shift to mobile network. Statistical report on the development of the Internet in China shows that denizens in China has reached 7.72 billion until 2017, the penetration rate in 2017 is $55.8 \%$, which increases $4.1 \%$ over 2015; the number of mobile internet users in 2017 is 7.53 billion, which increases about 63 million over 2016. Surfing internet though mobile has been a working or studying habit for users.

The role of intelligence has been great promoted by the widely using applications of big data. In big data era, data can be generated in every domain and applications and gradually become one of important productive factors. The bid data application plays the fundamental role in the future competition. The report in IBM indicates that $58 \%$ of companies has implemented data analysis techniques into market, obtained advantages for competition and achieved the business worth, which increases $21 \%$ comparing with that of previous years. The modification of decision making pattern implies the high value of intelligence ${ }^{[9]}$.

\section{The Strategies for CI in Big Data Era}

The advent of the era of big data has no doubt that the data has become an important asset to change the mode of enterprise decision-making. Enterprise competitive intelligence workers need to face the challenge of the change, change ideas with all possible means to fully tap the value of data, for enterprises to create sustainable competitive advantage to provide intellectual support.

\subsection{Establish intelligence consciousness based on big data}

Despite the rapid growth of large data and the development of related technologies are bringing new business opportunities, but there are still a lot of people on big data and the existence of the value of understanding is not clear enough. According to a survey, currently $49 \%$ of organizations are very concerned about the management of big data topic, but $38 \%$ of respondents do not understand what is big data, and another $27 \%$ of people said their understanding of more than one sided. Particularly fatal is that most small and medium enterprises believe that big data is Google, Amazon, Facebook, Alibaba, such as other companies are concerned about the technology. More people believe that big data is just a gimmick to attract the eye of business, the information explosion brought about by this phenomenon has been existed. Essence, big data is speed and amount of data generated from beyond the people data processing capability and is pregnant with a new concept, is a sign from quantitative change to qualitative change data. The rise of big data, is prompting companies to look at data strategies, hoping to dig more business value from big data analysis. The fact also shows that the use of big data is becoming a leading enterprise in the performance of an important way to go beyond their peers.

\subsection{The ability to set up a large data analysis of the competitive intelligence team}

Big data can be converted into actionable intelligence is a prerequisite for large data analysis capabilities. From the original data to the refining process of competitive intelligence is not only the challenge of IT technical staff, but also a challenge to industry experts, because the relationship between data has not entirely technical problems, some association only professionals can know must be in sales, finance, logistics and other aspects of professional staff, and even the need for ecologists, mathematics and statisticians, social network experts, social behavioural psychologists expert help and analytical, in order to establish a reasonable data structure. That is to say, the future of intelligence analysis requires the cooperation of IT technicians and industry experts ${ }^{[10]}$. Therefore, the need to adopt a flexible strategy to build large data related to human resources. Such as:

(1) Strengthen technical training. Traditional query, retrieval and reporting methods are difficult 
to adapt to the requirements of the era of big data, for a lot of competition intelligence personnel, it is urgent to introduce more specific technical training, training such as Hadoop, MapReduce and NoSQL and big data platform, let them familiar with the method and technology for the next generation of professional knowledge; strengthen the statistics and Analysis on training and mastering in big data platform in intelligence analysis of the theories, methods and tools.

(2) Employment outsourcing. In order to reduce the scale of the competitive intelligence team and the cost of human resources, outsourcing can be used to outsource some big data analysis to other companies. This method is suitable for task based.

(3) Colleges and universities training. Huge talent gap, the need to find a solution from the source. Colleges and universities is the main channel of our country at present, therefore, we should adapt to the demand of big data, strengthen the teaching of big data analysis.

From a technical point of view, construct the enterprise competitive intelligence system based on cloud computing, enterprise competitive intelligence system to realize the data processing, in addition to make full use of the MapReduce, NoSQL, Hadoop and other big data technology and infrastructure need to meet the following points:

(1) Capacity is large enough, can accommodate PB level data; (2) Strong analysis ability, with integrated analysis to accelerate advanced modelling and analysis of operation process; (3) Fast response to support low latency data access and decision. Which makes the enterprises great pressure of cost in hardware and software, and the increasing.

Therefore, the enterprise must to consider the feasibility and cost of using the data to re-examine the construction strategy of competitive intelligence system. Cloud computing is a kind of distributed computing, grid computing, parallel computing and the Internet combine new IT resources provide mode, can realize it resources, automated management and configuration, reduce the complexity of the IT management, improve the efficiency of resource use. Cloud computing has three notable features, such as "resource sharing, quick delivery, on-demand service".

\subsection{Strengthening the construction of information security system}

Information security is not only a technical problem, but also a management problem. Therefore, large data environment, enterprise in addition to technically realize anti hacker, anti-virus, and to defend against the threat of alien invaders, more need to focus on strengthening in information security system, information resource sharing system, the protection of confidential information, information audit system construction, from the management to eliminate enterprise core business data and business secrets have been leaked vulnerabilities ${ }^{[10]}$.

(1) Information security assurance system. In accordance with the relevant national information security technology standards, the establishment of enterprise information security risk assessment procedures and norms; according to the standard of information security ISO27001, with the establishment of the national information security information security system; to protect the safety of the system as a benchmark, the establishment of the organization of enterprise information security; establish daily safety operation and maintenance mechanism, including the safe operation of the supervision control and deal with the problem, and change management; rapid emergency response system, including data disaster backup, various business systems and it systems of emergency response plan.

(2) Information resource sharing system. Here mainly discusses the information resources sharing security measures. Mainly including: the establishment of enterprise information security, to define each categories of information dissemination scope; to develop enterprise information storage, transfer, copy and other provisions; for storage in the cloud data, according to the level of importance of information, the implementation of information "in the cloud" storage ${ }^{[10]}$.

(3) Confidential information protection system. Establish critical information recognition mechanism, regularly update the category of secret information in enterprise; enterprise security areas, reduce the confidential information of the contact and communication link; encrypt the confidential information; storage of confidential information safety regulations are formulated, including security, fire prevention, waterproof; set up exclusion protocol, prevent classified staff 
and senior managers after the departure of leak; confidentiality provisions are formulated to prevent consultancy, cloud service providers third-party leaks.

(4) Information audit system. Constructing prevention and unified security monitoring and auditing information disclosure of information audit process; establishment of information audit and monitoring working group, responsible for safety monitoring and assessment of the enterprise information resource system; determine the scope of the audit information, develop information audit cycle, on a regular basis for safety assessment and user operate; for cloud leak, can introduce third party information security audit mechanism on cloud data deposit regular risk assessment.

\section{Conclusion}

The advent of the era of big data has no doubt. Big data has the characteristics of large amount of data, many types, sparse value and fast speed, which brings opportunities and challenges to enterprise competitive intelligence work. Enterprise competitive intelligence. We must face these opportunities and challenges in the future development process, only those enterprises that can use these new data types can create sustainable competitive advantage. The on the basis of detailed data of the concept, characteristics, in-depth analysis of the data to the competitive intelligence work brings opportunities and challenges, and accordingly the future enterprise competitive intelligence work coping strategies were predicted.

\section{References}

[1] AE Haddadi., A Fennan., B Dousset and Z Boulouard, "XEW 2.0: A Competitive Intelligence System for Big Data Analysis in Cloud,” International Journal of Latest Research in Science \& Technology, 2017, 03(11), 19-25.

[2] X Huang, H Zhong, "The Construction of the Competitive Intelligence System in Small and Medium-Sized Enterprises in a Cloud Environment”, Information \& Documentation Services, 2012, 84(4), 39-43.

[3] W Zhang, "Research on Competitive Intelligence Security Problems and Strategies in Cloud Computing Era”, Journal of Intelligence, 2011, 30(7), 8-12.

[4] Meng-ru Li, Ruo-dan Sun, Hong Fu, Shi-tian Shen. Competetive intelligence changes in big data era based on literature analysis 2016 3rd international conference on economic and management, 2016, 36-40.

[5] Huang Xiaobin, Zhong Huixin. On the Innovation and Development of Enterprises Competitive Intelligence Analysis in the Big-data Era. Library and Information, 2012, 6, 9-14.

[6] Yang Y, Wu M. Z., Cui L. Integration of three visualization method based on co-word analysis. Scientometrics, 2012, 2, 659-673.

[7] Liu Pengrui, Wang Xu. Analysis on Hotspots of Competitive Intelligence of China in the Big Data Environment. Library Sciences Research \& Work, 2017, 3, 25-28.

[8] Jing Di, Bo He, Wen Li. Research on Enterprise Competitive Intelligence Development and Strategis in the Big Data Era. IEEE ICIT Proceedings, 2014, 658-663.

[9] Wu Jinhong, Wang Cuibo. Research on Evaluation Index System of Competitive Intelligent System in Big Data Era. Journal of Modern Information, 2016, 2, 3-7.

[10] Wu Jinhong, Zhang Fei, Ju Xiufang. Big Data: Opportunities, Challenges and Strategies of Enterprise Competitive Intelligence. Journal of Information, 2013, 1, 5-9. 